\title{
雷酸家族史话
}

王常清 ${ }^{*}$, 曹小华, 钟婵娟, 王建国, 严平

九江学院化学与环境工程学院, 江西 九江 332005

摘要: 通过雷酸自我介绍的方式来介绍雷酸及雷酸盐的发现、结构的解析以及在社会生活中的应用。从早期炼金术 士发现雷酸录, 到结构分析中同分异构体概念的提出, 再到诺贝尔采用雷酸录研制炸药的成功, 雷酸家族的故事为 科学与技术彼此交融、共同进步提供了一个很好的例子。

关键词: 雷酸; 雷酸录; 同分异构体; 炸药

中图分类号: G64; O6

\section{The Story of Fulminic Acid and Fulminates}

Changqing Wang ", Xiaohua Cao, Chanjuan Zhong, Jianguo Wang, Ping Yan

College of Chemistry and Environment Engineering, Jiujiang University, Jiujiang 332005, Jiangxi Province, P. R. China.

Abstract: This article describes an introduction of fulminic acid. The story of fulminic acid and fulminates was introduced from the discovery of mercury fulminate to the concept of isomers and the success of Alfred Nobel in the development of explosives. This process provides a good example of integrating science and technology.

Key Words: Fulminic acid; Mercury fulminate; Isomer; Explosive

大家好! 我是雷酸, 我的英文名字叫 fulminic acid, HCNO 是我的分子式。今天我来给大家介绍 我们的家族, 很多人对我可能并不熟悉, 但提到我的大哥—一雷酸永, 那可真是响当当的一条好汉, 当年诺贝尔研制炸药, 正是用它作起爆药剂, 开山䢃石的雷管以它得名。忘了介绍, 雷酸录的英文 名叫 mercury fulminate, 江湖上行走, 大家可能还会见到各种 fulminating $\mathrm{xxx}$ 名号的暴脾气们, 请 别搞混了, 它们的中文名字叫雷爆盐, 与我们完全不是一个家族 ${ }^{[1]}$ 。读到这里, 您或许会感到有些困 惑, 请别急, 下面且听我一一道来。

\section{1 雷酸家族的发现}

我们家族的历史非常悠久, 首先被发现的还是大哥雷酸录。原因很简单，当时西方的炼金术士 们认为永是一切金属的化身, 经常变着花样地折腾含录化合物, 同时雷酸录性格火爆, 受不得半点 刺激。早在公元 14 世纪荷兰人德雷贝尔就已经听到了它的怒吼, 或许是出于保密的原因, 这位老先 生的记录有些语焉不详 ${ }^{[2]}$ 。对雷酸录第一次详细描绘是 15 世纪的德国人昆克尔 ${ }^{[3]}$, 他将银和录溶于 硝酸之中, 再加入酒精, 反应非常剧烈, 随后发生的巨大爆炸吓得人们心胆俱裂, 还以为是魔鬼降 临。从留下的实验记录可以推测, 这位老兄无意间制备出了雷酸录, 也见识了它的爆脾气, 但是除 了满屋狼藉, 他并没有真正得到雷酸录。第一次真正获得雷酸录的是英国化学家霍华德。1800 年,

收稿: 2020-06-01; 录用: 2020-07-20; 网络发表: 2020-07-27

“通讯作者, Email: pharmawang@163.com

基金资助：江西省教育厅教改课题(JXJG-18-17-8) 
霍华德准备用氧化录为碱, 硝酸和酒精为氢和氧的供体, 试图合成当时学界臆想中的一种 “氯酸” 化合物 ${ }^{[3]}$ 。从现代化学的角度来看, 不得不说他的这一番操作真是难以描述。结果反应生成了大量 的白色晶体, 当霍华德取出一部分样品用浓硫酸处理的时候, 新化合物 “轰” 的一声拂袖而去的场 景吓得他差点怀疑人生。不用说, 这些狂暴的白色晶体就是我们的带头大哥—一雷酸录。由于它爆 炸的表现大大超过了当时的火药, 霍华德对此非常感兴趣。他仔细研究了诱发雷酸录爆炸的各种条 件, 以及爆炸产生的威力等详细参数, 还与克鲁克香克合作, 合成了我的二哥雷酸银, 要知道雷酸 银的脾气可比雷酸录坏多了, 它甚至能被自身的重量引爆 ${ }^{[4]}$ 。一时间霍华德的实验室爆炸声此起彼 伏, 轰隆隆的爆鸣声给霍华德带来了名声, 以及他梦棩以求的皇家学会科普利奖章, 也给他的身体 造成了严重的伤害。但是这些谜之白色晶体为何如此暴躁？它们的结构到底是什么样子? 由于当时 有机定量分析的方法还没有建立, 化学理论的发展也比较初级, 对这些问题, 霍华德深感力所不逮, 带着满身的伤痕黯然离去。

\section{2 雷酸家族的结构}

时间到了 1823 年, 后来被誉为 “有机化学之父” 的李比希在导师盖·吕萨克的实验室里对雷酸 银进行了仔细的分析, 他意外地发现其成分与同时期维勒测定的氰酸银的组成居然完全相同, 这怎 么可能? 雷酸银性情火爆, 氰酸银安静沉稳, 两者的性格天差地别, 化学组成居然一样? 李比希立 即联系维勒, 质疑他的实验数据。两人重新验证了分析结果, 发现双方的测定数据都准确无误。这 个发现让他们百思不得其解, 因为当时化学界普遍认为只有当元素组成不同的化合物性质才会不一 样。为了解释这种现象, 维勒的导师——瑞典化学家贝采利乌斯在 1830 年提出了同分异构的概念: 同样的化学成分, 可以组成性质不同的化合物。同分异构现象让化学家们意识到, 研究物质不仅是 测出其组成就可以了, 还需要研究分子的结构。雷酸银和氧酸银也由此成为人类最早发现的同分异 构体。不打不相识, 正是由于研究雷酸盐成分的机缘, 李比希和维勒原本素不相识的两位青年人, 开始了长达 44 年亲密无间的交往, 最终成为有机化学界的两大巨擘。有机化学能发展到今天, 我们 雷酸家族真是功不可没啊!

根据李比希的分析, 我的分子组成极其简单, 其中氢碳氮氧的比例为 $1: 1: 1: 1$ 。但是各原子 如何组合成雷酸的分子? 这个看似很简单的问题, 却是让科学家们伤透了脑筋。因为雷酸是由乙醇 在硝酸存在下转化而来的, 而且常常以二元酸的形式存在, 所以最初大家推测我的结构是含有两个 碳的分子。比如 1857 年凯库勒认为我长这个样子: $\mathrm{CH}_{2} \mathrm{NO}_{2} \mathrm{CN}$; 格里斯看到我们家族成员个个性格 泼辣, 根据自己研究的重氮化合物易爆的经验, 推断我的结构是 $\mathrm{N}_{2} \mathrm{CHCOOH}$, 除此之外, 还有很多 奇奇怪怪的结构 ${ }^{[5]}$ 。直到 1882 年埃伦贝格和施泰纳分别通过实验确定雷酸盐通过酸水解定量地裂解 为羟胺和甲酸, 科学界才逐渐意识到: 原来二元酸是二聚的结果, 雷酸本身只含有一个碳, 这可真 是太雷了！1894 年, 美国化学家奈夫提出了一种二价碳的肜结构 ${ }^{[6]}$, 认为我长成这样: $\mathrm{C}=\mathrm{NOH}$, 这个结构一度在各种假说中占了上风, 但它很快就受到了腈氧化物 HCNO 结构的挑战(图 1)。由于 肜式结构需要引入二价碳的概念, 而腈氧化物则包含五价氮的结构, 都不符合最外层 8 电子的稳定 结构。随着价键理论的发展, 科学家们推出共振结构概念, 肜式结构 1 能够以 $1 \mathbf{a}$ 的结构, 腈氧化物 2 可以 $2 \mathbf{a}$ 或 $2 \mathbf{b}$ 或者是两种结构的共振杂化体存在。双方各有拥歪, 争论不休, 比如两次获得诺贝 尔奖的鲍林在 1926 年根据自由能计算出结构 $\mathbf{2}$ 的能量比 $\mathbf{1}$ 要更低, 但由于缺乏实验数据支持, 这个 论据并没有得到广泛的认可。以至于在很长一段时间里, 我的真正结构成了一段悬而未决的公案。

直到 1965 年, 德国科学家贝克第一次制备出纯的气态雷酸分子, 并利用红外光谱进行分析 ${ }^{[7],}$ 发现分子中并没有 $\mathrm{O}-\mathrm{H}$ 键的伸缩振动 (大约在 $3600 \mathrm{~cm}^{-1}$ 附近), 而只能观察到 $\mathrm{C}-\mathrm{H}$ 键的伸缩振动 $3335 \mathrm{~cm}^{-1}$, 以及 $\mathrm{HCN}$ 的弯曲振动等数据, 终于一锤定音确定了我的结构为腈的氮氧化合物形式(表 1)。至此, 在被发现一百多年以后, 关于我结构的争论终于尘埃落定。 


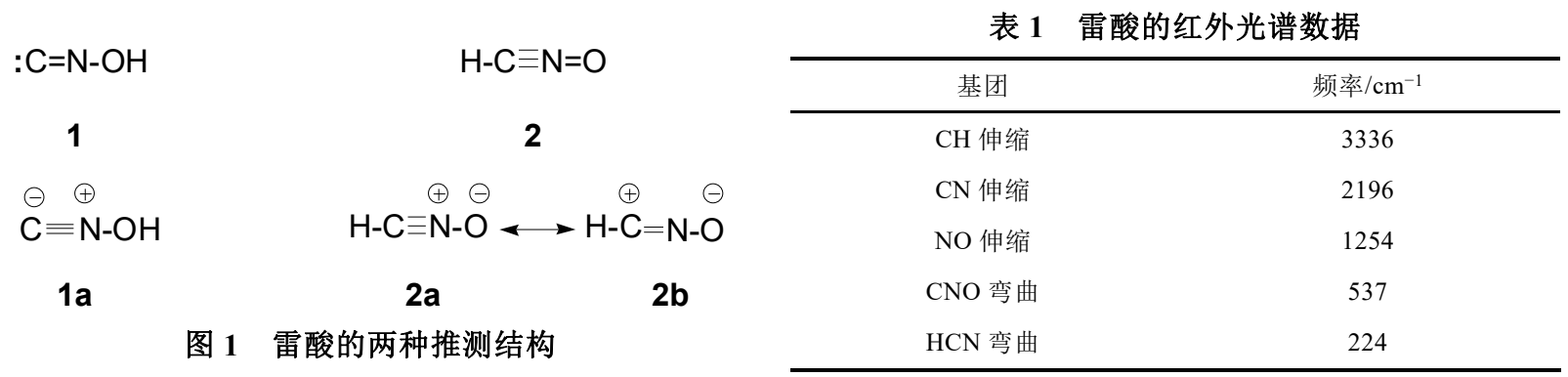

2007 年, 贝克又用 $\mathrm{X}$ 射线测出了雷酸录的晶体结构 ${ }^{[2]}$, 在原子尺度的层面获得其精确的结构信 息, 终于为我们家族准确的肖像画上了圆满的句号(图 2)。



图 2 雷酸泉的 $\mathbf{X}$ 单晶衍射图

红、蓝、黑及灰色分別代表氧、氮、碳及录原子 电子版为彩图

谈到这里, 大家应该很容易把雷爆盐与我们区别清楚了。比如制备银氨溶液时, 硝酸银中加入 氨水, 长时间放置或加热可能会生成的爆炸物就是雷爆盐。原因很简单, 配置银氨溶液的原料中不 含碳，生成雷酸银当然无从谈起，他们的结构更可能是氮化银 $\left(\mathrm{Ag}_{3} \mathrm{~N}\right)$ 、亚氨基化银 $\left(\mathrm{Ag}_{2} \mathrm{NH}\right)$ 。

\section{3 雷酸家族的制备}

在当年霍华德制备 “氯酸” 的实验中, 硝酸首先氧化乙醇生成乙醛, 乙醛在微量的亚硝酸存在 下亚硝化为肜基乙醛, 再被硝酸氧化为肜基乙酸, 该化合物与硝酸进一步发生硝化反应并经历脱羧、 脱亚硝酸过程即可生成雷酸。实际上生成的雷酸在此溶液中并不稳定, 但是由于溶液中同时存在的 采离子立即与之反应生成溶解度较低的雷酸录, 从而形成沉淀脱离了反应体系(图 3) ${ }^{[8]}$ 。上述反应的 步骤虽然复杂，但是产率却是相当高。回溯历史，当年霍华德无疑是幸运的，在众多的金属元素中， 除了录和银, 该反应都不会成功(录和银一方面可以与硝酸生成催化量的亚硝酸, 另一方面可以与雷 酸反应生成溶剂度较低的雷酸盐沉淀)。冥冥之中, 霍华德选择了最正确的打开方式, 吒开了雷酸家 族的大门。时至今日, 该法仍然是制备雷酸录的标准方法, 以录盐为原料, 我们其余大部分的家族 成员都可以得到了。



图 3 雷酸采的制备原理

\section{4 雷酸家族的性质与应用}

俗话说: 性格决定命运, 对于化合物来说, 那就是结构决定性质。从结构来看我们是一种含三 
原子、四电子体系的 1,3-偶极化合物, 而且也是一大类含氧化腈官能团化合物的母体。这种结构可 以发生很多反应，其中最有名的当属惠斯根发明的 1,3-偶极环加成反应，他发现我们可以和烯烃、 炔烃或其衍生物之间发生环加成反应生成异噁唑啉化合物(图 4) ${ }^{[9]}$ 。由于含有该片段的化合物很多 具有较好的生物活性, 而被广泛应用于除草、杀虫、抗病毒等领域, 比如年销售额到达 1.3 亿美元 的明星除草剂药物一一砜吡草唑, 就是一个含异啞唑啉官能团的化合物。因此, 高效地构建异噁唑啉 环对此类药物开发的意义是不言而喻的, 而我们的结构恰好就能一步环化生成异啞唑啉环, 说到这 里，雷酸心中满满的都是自豪啊。
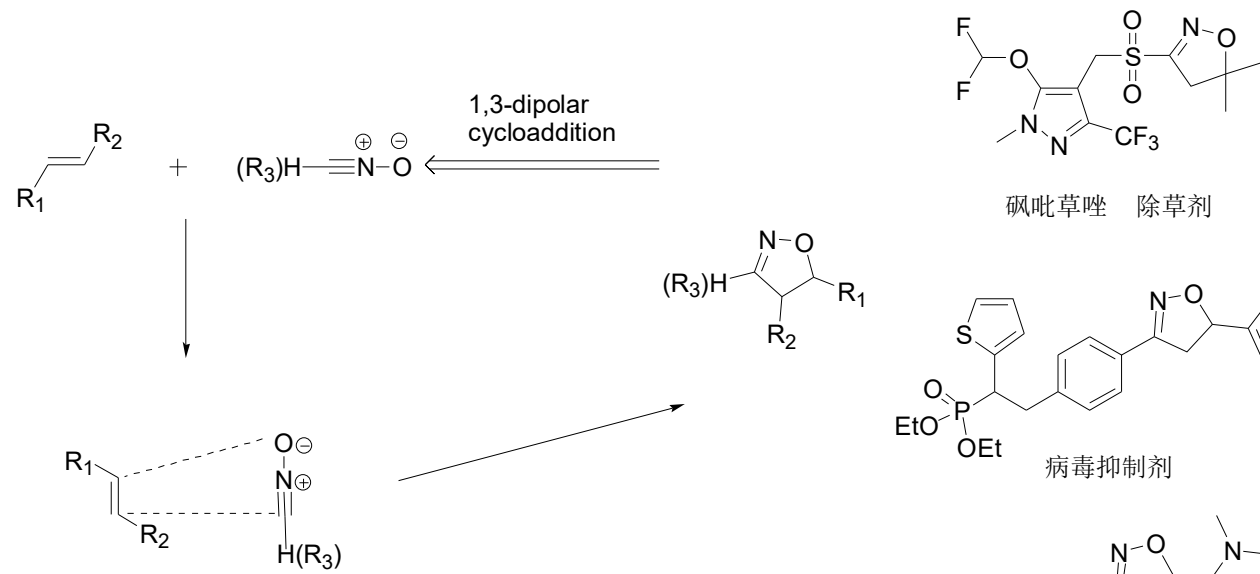

砜吡草唑 除草剂
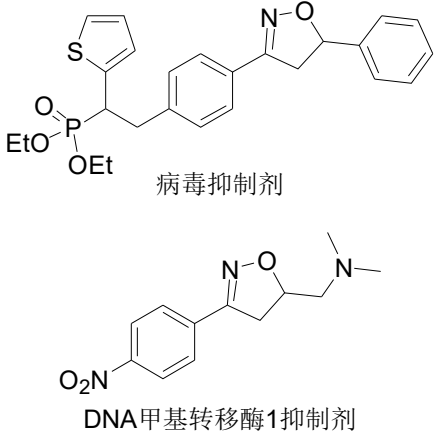

图 4 雷酸及衍生物发生 $1,3-$ 偶极环加成反应

除了在药物研发方面的应用, 我们在工业领域也颇有建树。由于家族成员普遍具有人狠话不多 的性格, 人们一开始希望可以用雷酸盐来代替火药。但是由于性格过于敏感, 这方面的种种尝试都 失败了。还好, 大哥雷酸录最终找到一个作引信的差事。在军用方面, 早期的火枪引信用的是氯化 钾混合物, 这些物质容易吸潮, 而且枪管也容易被腐蚀, 雷酸录较好地解决了这些问题; 在民用方 面, 诺贝尔发现硝化甘油混入多孔材料后变得很稳定, 但是这种炸药很难被引爆, 直到采用雷酸永 作引信, 他日思夜想的安全而爆炸力强烈的炸药才终于被研制出来, 这也标志着人类进入到了一个 安全爆炸的新时代。

以上就是关于我们雷酸家族的故事，从炼金术士到同分异构体，再到诺贝尔的成功，人类在认 识自然的过程中, 科学与技术的发展彼此包含, 相互促进, 共同进步, 我们的故事提供了一个很好 的例子。

\section{参 考 文 献}

[1] 伊恩·詹金斯, 李雄记. 化学教育, 1990, 11 (6), 48.

[2] Beck, W.; Evers, J.; Gobel, M.; Oehlinger, G.; Klapotk, T. M. Z. Anorg. Allg. Chem. 2007, 633 (9), 1417.

[3] Kurzer, F. J. Chem. Edu. 2000, 77 (7), 851.

[4] Kurzer, F. Ann. Sci. 1999, 56, 113.

[5] Kekulé, A. Ann. Chem. 1857, 101, 200.

[6] Nef, J. U. Ann. Chem. 1894, 280, 291.

[7] Beck, W.; Feldl, K. Angew. Chem. Int. Ed. 1966, 5 (8), 722.

[8] Wieland, H. Ber. Deutsch. Chem. Ges. 1907, 40, 418.

[9] Huisgen, R.; Christl, M. Angew. Chem. Int. Ed. 1967, 6 (5), 456. 